\title{
An Isolated Bipolar Single-Valued Neutrosophic Graphs
}

\author{
Said Broumi ${ }^{1(\bowtie)}$, Assia Bakali ${ }^{2}$, Mohamed Talea $^{1}$, \\ and Florentin Smarandache ${ }^{3}$ \\ ${ }^{1}$ Laboratory of Information Processing, Faculty of Science Ben M'Sik, \\ University Hassan II, Casablanca, Morocco \\ broumisaid78@gmail.com, taleamohamed@yahoo.fr \\ 2 Ecole Royale Navale, Boulevard Sour Jdid, B.P 16303 Casablanca, Morocco \\ assiabakali@yahoo.fr \\ ${ }^{3}$ Department of Mathematics, University of New Mexico, 705 Gurley Avenue, \\ Gallup NM87301, USA \\ fsmarandache@gmail.com
}

\begin{abstract}
In this research paper, we propose the graph of the bipolar single-valued neutrosophic set (BSVNS) model. This graph generalized the graphs of single-valued neutrosophic set models. Several results have been proved on complete and isolated graphs for the BSVNS model. Moreover, an essential and satisfactory condition for the graphs of the BSVNS model to become an isolated graph of the BSVNS model has been demonstrated.
\end{abstract}

Keywords: BSVNGs $\cdot$ Complete BSVNG $\cdot$ Isolated BSVNGs

\section{Introduction}

Smarandache [1] proposed the concept of neutrosophic sets (in short NSs) as a means of expressing the inconsistencies and indeterminacies that exist in most real-life problems. The proposed concept generalized fuzzy sets and intuitionistic fuzzy sets theory [2, 3]. The notion of NS is described with three functions: truth, an indeterminacy and a falsity, where the functions are totally independent; the three functions are inside the unit interval $]^{-} 0,1^{+}[$. To practice NSs in real-life situations efficiently, a new version of NSs. A new version of NSs named single-valued neutrosophic sets (in short SVNSs) was defined by Smarandache in [1]. Subsequently, Wang et al. [4] defined the various operations and operators for the SVNS model. In [5], Deli et al. introduced the notion of bipolar neutrosophic sets, which combine the bipolar fuzzy sets and SVNS models. Neutrosophic sets and their extensions have been paid great attention recent years [6]. The theory of graphs is the mostly used tool for resolving combinatorial problems in various fields such as computer science, algebra and topology. Smarandache [1, 7] introduced two classes of neutrosophic graphs to deal with situations in which there exist inconsistencies and indeterminacies among the vertices which cannot be dealt with by fuzzy graphs and different hybrid structures [810]. The first class is relied on literal indeterminacy (I) component, and the second class of neutrosophic graphs is based on numerical truth values (T, I, F). Subsequently, 
Broumi et al. [11-13] introduced the concept single-valued neutrosophic graphs (in short SVNGs) and discussed some interesting results. Later on, the same authors [1417] proposed the concept of bipolar single-valued neutrosophic graphs (BSVNGs) and established some interesting results with proofs and illustrations.

The objective of our article is to demonstrate the essential and satisfactory condition of BSVNGs to be an isolated BSVNG.

\section{Background of Research}

Some of the important background knowledge for the materials that are presented in this paper is presented in this section. These results can be found in $[1,4,5,12,13]$.

Definition 2.1 [1]. Let $\zeta$ be a universal set. The neutrosophic set $A$ on the universal set $\zeta$ is categorized into three membership functions, namely the true $T_{A}(\mathrm{x})$, indeterminate $I_{A}(\mathrm{x})$ and false $F_{A}(\mathrm{x})$ contained in real standard or non-standard subset of $]^{-} 0,1^{+}$, respectively.

$$
{ }^{-} 0 \leq \sup \mathrm{T}_{\mathrm{A}}(\mathrm{x})+\sup \mathrm{I}_{\mathrm{A}}(\mathrm{x})+\sup \mathrm{F}_{\mathrm{A}}(\mathrm{x}) \leq 3^{+}
$$

Definition 2.2 [4]. Let $\zeta$ be a universal set. The single-valued neutrosophic sets (SVNSs) A on the universal $\zeta$ is denoted as following

$$
\mathrm{A}=\left\{<x: \mathrm{T}_{\mathrm{A}}(\mathrm{x}), \mathrm{I}_{\mathrm{A}}(\mathrm{x}), \mathrm{F}_{\mathrm{A}}(\mathrm{x})>\mathrm{x} \in \zeta\right\}
$$

The functions $\mathrm{T}_{\mathrm{A}}(\mathrm{x}) \in[0,1], \mathrm{I}_{\mathrm{A}}(\mathrm{x}) \in[0,1]$ and $\mathrm{F}_{\mathrm{A}}(\mathrm{x}) \in[0,1]$ are called "degree of truth, indeterminacy and falsity membership of $\mathrm{x}$ in $\mathrm{A}$ ", which satisfy the following condition:

$$
0 \leq T_{A}(\mathrm{x})+I_{A}(\mathrm{x})+F_{A}(\mathrm{x}) \leq 3
$$

Definition 2.3 [12]. A SVNG of $G^{*}=(\mathrm{V}, \mathrm{E})$ is a graph $\mathrm{G}=(\mathrm{A}, \mathrm{B})$ where

a. The following memberships: $T_{A}: \mathrm{V} \rightarrow[0,1], \mathrm{I}_{\mathrm{A}}: \mathrm{V} \rightarrow[0,1]$ and $\mathrm{F}_{\mathrm{A}}: \mathrm{V} \rightarrow[0,1]$ represent the truth, indeterminate and false membership degrees of $x \in V$ respectively and

$$
0 \leq T_{A}(w)+I_{A}(w)+F_{A}(w) \leq 3 \quad \forall \mathrm{w} \in \mathrm{V}
$$

b. The following memberships: $T_{B}: \mathrm{E} \rightarrow[0,1], I_{B}: \mathrm{E} \rightarrow[0,1]$ and $\mathrm{F}_{\mathrm{B}}: \mathrm{E} \rightarrow[0,1]$ are defined by

$$
\begin{gathered}
\mathrm{T}_{\mathrm{B}}(v, w) \leq \min \left[\mathrm{T}_{\mathrm{A}}(v), \mathrm{T}_{\mathrm{A}}(w)\right] \\
I_{B}(v, w) \geq \max \left[\mathrm{I}_{\mathrm{A}}(v), \mathrm{I}_{\mathrm{A}}(w)\right] \text { and }
\end{gathered}
$$




$$
F_{B}(v, w) \geq \max \left[\mathrm{F}_{\mathrm{A}}(v), \mathrm{F}_{\mathrm{A}}(w)\right]
$$

Represent the true, indeterminate and false membership degrees of the $\operatorname{arc}(v, w) \in$ (V x V), where (Fig. 1)

$$
0 \leq \mathrm{T}_{\mathrm{B}}(v, w)+\mathrm{I}_{\mathrm{B}}(v, w)+\mathrm{F}_{\mathrm{B}}(v, w) \leq 3 \quad \forall(v, w) \in \mathrm{E}
$$

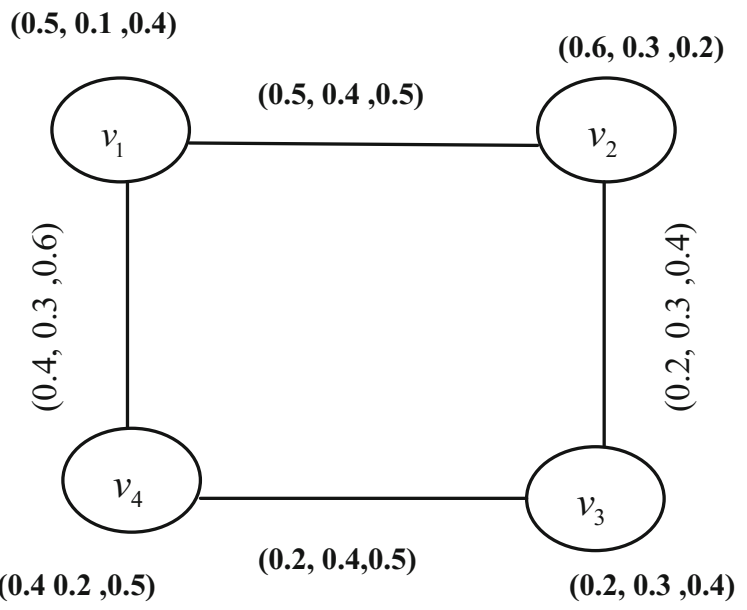

Fig. 1. SVN graph

Definition 2.4 [12]. A SVNG G = (A, B) is named complete SVNG if

$$
\begin{gathered}
\mathrm{T}_{\mathrm{B}}(v, w)=\min \left[\mathrm{T}_{\mathrm{A}}(\mathrm{v}), \mathrm{T}_{\mathrm{A}}(w)\right] \\
\mathrm{I}_{\mathrm{B}}(v, w)=\max \left[\mathrm{I}_{\mathrm{A}}(\mathrm{v}), \mathrm{I}_{\mathrm{A}}(w)\right] \\
\mathrm{F}_{\mathrm{B}}(v, w)=\max \left[\mathrm{F}_{\mathrm{A}}(\mathrm{v}), \mathrm{F}_{\mathrm{A}}(w)\right] \quad \forall v, w \in \mathrm{V}
\end{gathered}
$$

Definition 2.5 [12]. Given a SVNG G = (A, B). Hence, the complement of SVNG on $G^{*}$ is a SVNG $\bar{G}$ on $G^{*}$ where

$$
\begin{gathered}
a \cdot \bar{A}=\mathrm{A} \\
b \cdot \bar{T}_{A}(w)=T_{A}(w), \bar{I}_{A}(w)=I_{A}(w), \bar{F}_{A}(w)=F_{A}(w) \quad \forall w \in \mathrm{V} \\
c \cdot \overline{\mathrm{T}}_{\mathrm{B}}(v, w)=\min \left[\mathrm{T}_{\mathrm{A}}(v), \mathrm{T}_{\mathrm{A}}(w)\right]-\mathrm{T}_{\mathrm{B}}(v, w)
\end{gathered}
$$




$$
\begin{gathered}
\overline{\mathrm{I}}_{\mathrm{B}}(v, w)=\max \left[I_{A}(v), I_{A}(w)\right]-I_{B}(v, w) \text { and } \\
\overline{\mathrm{F}}_{\mathrm{B}}(v, w)=\max \left[F_{A}(v), F_{A}(w)\right]-F_{B}(v, w), \forall(v, w) \in \mathrm{E}
\end{gathered}
$$

Definition 2.6 [14]. A BSVNG of $G^{*}=(\mathrm{V}, \mathrm{E})$ is a partner $G=(\mathrm{A}, \mathrm{B})$ where $\mathrm{A}=$ $\left(\mathrm{T}_{\mathrm{A}}^{\mathrm{P}}, \mathrm{I}_{\mathrm{A}}^{\mathrm{P}}, \mathrm{F}_{\mathrm{A}}^{\mathrm{P}}, \mathrm{T}_{\mathrm{A}}^{\mathrm{N}}, \mathrm{I}_{\mathrm{A}}^{\mathrm{N}}, \mathrm{F}_{\mathrm{A}}^{\mathrm{N}}\right)$ is a BSVNS in $\mathrm{V}$ and $\mathrm{B}=\left(\mathrm{T}_{\mathrm{B}}^{\mathrm{P}}, \mathrm{I}_{\mathrm{B}}^{\mathrm{P}}, \mathrm{F}_{\mathrm{B}}^{\mathrm{P}}, \mathrm{T}_{\mathrm{B}}^{\mathrm{N}}, \mathrm{I}_{\mathrm{B}}^{\mathrm{N}}, \mathrm{F}_{\mathrm{B}}^{\mathrm{N}}\right)$ is a BSVNS in $\tilde{\mathrm{V}}^{2}$ such that (Fig. 2)

$$
\begin{aligned}
& \mathrm{T}_{\mathrm{B}}^{\mathrm{P}}(v, w) \leq \min \left(\mathrm{T}_{\mathrm{A}}^{\mathrm{P}}(v), \mathrm{T}_{\mathrm{A}}^{\mathrm{P}}(w)\right), \quad \mathrm{T}_{\mathrm{B}}^{\mathrm{N}}(v, w) \geq \max \left(\mathrm{T}_{\mathrm{A}}^{\mathrm{N}}(v), \mathrm{T}_{\mathrm{A}}^{\mathrm{N}}(w)\right) \\
& \mathrm{I}_{\mathrm{B}}^{\mathrm{P}}(v, w) \geq \max \left(\mathrm{I}_{\mathrm{A}}^{\mathrm{P}}(v), \mathrm{I}_{\mathrm{A}}^{\mathrm{P}}(w)\right) \quad \mathrm{I}_{\mathrm{B}}^{\mathrm{N}}(v, w) \leq \min \left(\mathrm{I}_{\mathrm{A}}^{\mathrm{N}}(v), \mathrm{I}_{\mathrm{A}}^{\mathrm{N}}(w)\right) \\
& \mathrm{F}_{\mathrm{B}}^{\mathrm{P}}(v, w) \geq \max \left(\mathrm{F}_{\mathrm{A}}^{\mathrm{P}}(v), \mathrm{F}_{\mathrm{A}}^{\mathrm{P}}(w)\right), \quad \mathrm{F}_{\mathrm{B}}^{\mathrm{N}}(v, w) \leq \min \left(\mathrm{F}_{\mathrm{A}}^{\mathrm{N}}(v), \mathrm{F}_{\mathrm{A}}^{\mathrm{N}}(w)\right) \quad \forall \mathrm{v}, \mathrm{w} \in \tilde{\mathrm{V}}^{2} \\
& v_{1}(0.2,0.2,0.4,-0.4,-0.1,-0.4) \\
& (0.1,0.3,0.6,-0.2,-0.3,-0.1) \\
& v_{2}(0.1,0.3,0.5,-0.6,-0.2,-0.3)
\end{aligned}
$$

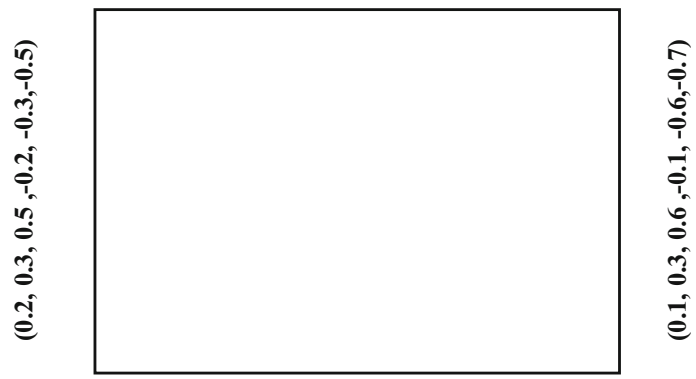

Fig. 2. BSVNG

Definition 2.7 [14]. The complement of BSVNG $\mathbf{G}=(A, B)$ of $G^{*}=(\mathrm{A}, \mathrm{B})$ is a BSVNG $\bar{G}=(\bar{A}, \bar{B})$ of $\bar{G}^{*}=(\mathrm{V}, \mathrm{V} \times \mathrm{V})$ where $\bar{A}=\mathrm{A}=\left(\mathrm{T}_{\mathrm{A}}^{\mathrm{P}}, \mathrm{I}_{\mathrm{A}}^{\mathrm{P}}, \mathrm{F}_{\mathrm{A}}^{\mathrm{P}}, \mathrm{T}_{\mathrm{A}}^{\mathrm{N}}, \mathrm{I}_{\mathrm{A}}^{\mathrm{N}}, \mathrm{F}_{\mathrm{A}}^{\mathrm{N}}\right)$ and $\overline{\mathrm{B}}=\left(\overline{\mathrm{T}}_{\mathrm{B}}^{\mathrm{P}}, \overline{\mathrm{I}}_{\mathrm{B}}^{\mathrm{P}}, \overline{\mathrm{F}}_{\mathrm{B}}^{\mathrm{P}}, \overline{\mathrm{T}}_{\mathrm{B}}^{\mathrm{N}}, \overline{\mathrm{I}}_{\mathrm{B}}^{\mathrm{N}}, \overline{\mathrm{F}}_{\mathrm{B}}^{\mathrm{N}}\right)$ is defined as

$$
\begin{gathered}
\overline{\mathrm{T}}_{\mathrm{B}}^{\mathrm{P}}(v, w)=\min \left(\mathrm{T}_{\mathrm{A}}^{\mathrm{P}}(v), \mathrm{T}_{\mathrm{A}}^{\mathrm{P}}(w)\right)-\mathrm{T}_{\mathrm{B}}^{\mathrm{P}}(v, w) \\
\overline{\mathrm{I}}_{\mathrm{B}}^{\mathrm{P}}(v, w)=\max \left(\mathrm{I}_{\mathrm{A}}^{\mathrm{P}}(v), \mathrm{I}_{\mathrm{A}}^{\mathrm{P}}(w)\right)-\mathrm{I}_{\mathrm{B}}^{\mathrm{P}}(v, w) \\
\overline{\mathrm{F}}_{\mathrm{B}}^{\mathrm{P}}(v, w)=\max \left(\mathrm{F}_{\mathrm{A}}^{\mathrm{P}}(v), \mathrm{F}_{\mathrm{A}}^{\mathrm{P}}(w)\right)-\mathrm{F}_{\mathrm{B}}^{\mathrm{P}}(v, w)
\end{gathered}
$$




$$
\begin{gathered}
\overline{\mathrm{T}}_{\mathrm{B}}^{\mathrm{N}}(v, w)=\max \left(\mathrm{T}_{\mathrm{A}}^{\mathrm{N}}(v), \mathrm{T}_{\mathrm{A}}^{\mathrm{N}}(w)\right)-\mathrm{T}_{\mathrm{B}}^{\mathrm{N}}(v, w) \\
\overline{\mathrm{I}}_{\mathrm{B}}^{\mathrm{N}}(v, w)=\min \left(\mathrm{I}_{\mathrm{A}}^{\mathrm{N}}(v), \mathrm{I}_{\mathrm{A}}^{\mathrm{N}}(w)\right)-\mathrm{I}_{\mathrm{B}}^{\mathrm{N}}(v, w) \\
\overline{\mathrm{F}}_{\mathrm{B}}^{\mathrm{N}}(v, w) \min \left(\mathrm{F}_{\mathrm{A}}^{\mathrm{N}}(v),(w)\right)-\mathrm{F}_{\mathrm{B}}^{\mathrm{N}}(v, w) \quad \forall v, w \in \mathrm{V}, v w \in \tilde{\mathrm{V}}^{2}
\end{gathered}
$$

Definition 2.8 [14]. A BSVNG $G=(A, B)$ is said to be complete BSVNG if

$$
\begin{array}{r}
\mathrm{T}_{\mathrm{B}}^{\mathrm{P}}(v, w)=\min \left(\mathrm{T}_{\mathrm{A}}^{\mathrm{P}}(v), \mathrm{T}_{\mathrm{A}}^{\mathrm{P}}(w)\right), \quad \mathrm{T}_{\mathrm{B}}^{\mathrm{N}}(v, w)=\max \left(\mathrm{T}_{\mathrm{A}}^{\mathrm{N}}(v), \mathrm{T}_{\mathrm{A}}^{\mathrm{N}}(w)\right), \\
\mathrm{I}_{\mathrm{B}}^{\mathrm{P}}(v, w)=\max \left(\mathrm{I}_{\mathrm{A}}^{\mathrm{P}}(v), \mathrm{I}_{\mathrm{A}}^{\mathrm{P}}(w)\right), \quad \mathrm{I}_{\mathrm{B}}^{\mathrm{N}}(v, w)=\min \left(\mathrm{I}_{\mathrm{A}}^{\mathrm{N}}(v), \mathrm{I}_{\mathrm{A}}^{\mathrm{N}}(w)\right) \\
\mathrm{F}_{\mathrm{B}}^{\mathrm{P}}(v, w)=\max \left(\mathrm{F}_{\mathrm{A}}^{\mathrm{P}}(v), \mathrm{F}_{\mathrm{A}}^{\mathrm{P}}(w)\right), \quad \mathrm{F}_{\mathrm{B}}^{\mathrm{N}}(v, w)=\min \left(\mathrm{F}_{\mathrm{A}}^{\mathrm{N}}(v), \mathrm{F}_{\mathrm{A}}^{\mathrm{N}}(w)\right) \quad \forall v, w \in \mathrm{V}
\end{array}
$$

Theorem 2.9 [13]: Let $\mathrm{G}=(\mathrm{A}, \mathrm{B})$ be a SVNG, then the SVNG is called an isolated SVNG if and only if the complement of $\mathrm{G}$ is a complete SVNG.

\section{Main Results}

Theorem 3.1: $\mathrm{A}$ BSVNG $=(\mathrm{A}, \mathrm{B})$ is an isolated BSVNG iff the complement of BSVNG is a complete BSVNG.

Proof: Given $G=(A, B)$ be a complete BSVNG.

$$
\begin{aligned}
& \operatorname{SoT}_{\mathrm{B}}^{\mathrm{P}}(v, w)=\min \left(\mathrm{T}_{\mathrm{A}}^{\mathrm{P}}(\mathrm{v}), \mathrm{T}_{\mathrm{A}}^{\mathrm{P}}(\mathrm{w})\right), \quad \mathrm{T}_{\mathrm{B}}^{\mathrm{n}}(v, w)=\max \left(\mathrm{T}_{\mathrm{A}}^{\mathrm{n}}(v), \mathrm{T}_{\mathrm{A}}^{\mathrm{n}}(w)\right), \\
& \mathrm{I}_{\mathrm{B}}^{\mathrm{P}}(v, w)=\max \left(\mathrm{I}_{\mathrm{A}}^{\mathrm{P}}(v), \mathrm{I}_{\mathrm{A}}^{\mathrm{P}}(w)\right), \quad \mathrm{I}_{\mathrm{B}}^{\mathrm{n}}(v, w)=\min \left(\mathrm{I}_{\mathrm{A}}^{\mathrm{n}}(v), \mathrm{I}_{\mathrm{A}}^{\mathrm{n}}(w)\right), \\
& \mathrm{F}_{\mathrm{B}}^{\mathrm{P}}(v, w)=\max \left(\mathrm{F}_{\mathrm{A}}^{\mathrm{P}}(v), \mathrm{F}_{\mathrm{A}}^{\mathrm{P}}(\mathrm{w})\right), \quad \mathrm{F}_{\mathrm{B}}^{\mathrm{n}}(v, w)=\min \left(\mathrm{F}_{\mathrm{A}}^{\mathrm{n}}(\mathrm{v}), \mathrm{F}_{\mathrm{A}}^{\mathrm{n}}(w)\right), \\
& \forall v, w \in V .
\end{aligned}
$$

Hence in $\overline{\mathrm{G}}$,

$$
\begin{aligned}
\bar{T}_{B}^{P}(v, w) & =\min \left(T_{A}^{P}(v), T_{A}^{P}(w)\right)-T_{B}^{P}(v, w) \\
& =\min \left(T_{A}^{P}(v), T_{A}^{P}(w)\right)-\min \left(T_{A}^{P}(v), T_{A}^{P}(w)\right) \\
& =0
\end{aligned}
$$

and

$$
\begin{aligned}
\bar{I}_{B}^{P}(\mathrm{v}, \mathrm{w}) & =\max \left(I_{A}^{P}(\mathrm{v}), I_{A}^{p}(\mathrm{w})\right)-I_{B}^{P}(\mathrm{v}, \mathrm{w}) \\
& =\max \left(I_{A}^{P}(\mathrm{v}), I_{A}^{P}(\mathrm{w})\right)-\max \left(I_{A}^{P}(\mathrm{v}), I_{A}^{P}(\mathrm{w})\right) \\
& =0
\end{aligned}
$$


In addition

$$
\begin{aligned}
\bar{F}_{B}^{P}(v, w) & =\max \left(F_{A}^{P}(v), F_{A}^{p}(w)\right)-F_{B}^{P}(v, w) \\
& =\max \left(F_{A}^{P}(v), F_{A}^{P}(w)\right)-\max \left(F_{A}^{P}(v), F_{A}^{P}(w)\right) \\
& =0
\end{aligned}
$$

We have for the negative membership edges

$$
\begin{aligned}
\bar{T}_{B}^{N}(v, w) & =\max \left(\mathrm{T}_{\mathrm{A}}^{\mathrm{N}}(v), \mathrm{T}_{\mathrm{A}}^{\mathrm{N}}(w)\right)-T_{B}^{N}(v, w) \\
& =\max \left(T_{A}^{N}(v), T_{A}^{N}(w)\right)-\max \left(\mathrm{T}_{\mathrm{A}}^{\mathrm{N}}(v), \mathrm{T}_{\mathrm{A}}^{\mathrm{N}}(w)\right) \\
& =0
\end{aligned}
$$

and

$$
\begin{aligned}
\bar{I}_{B}^{N}(v, w) & =\min \left(I_{A}^{N}(v), I_{A}^{N}(w)\right)-I_{B}^{N}(v, w) \\
& =\min \left(I_{A}^{N}(v), I_{A}^{N}(w)\right)-\min \left(I_{A}^{N}(\mathrm{v}), I_{A}^{N}(\mathrm{w})\right) \\
& =0
\end{aligned}
$$

In addition

$$
\begin{aligned}
& \bar{F}_{B}^{N}(v, w)=\min \left(F_{A}^{N}(v), F_{A}^{N}(w)\right)-F_{B}^{N}(v, w) \\
& =\min \left(F_{A}^{N}(v), F_{A}^{N}(w)\right)-\min \left(F_{A}^{N}(v), F_{A}^{N}(w)\right) \\
& =0
\end{aligned}
$$

So $\left(\bar{T}_{B}^{P}(v, w), \overline{\mathrm{I}}_{\mathrm{B}}^{\mathrm{p}}(v, w), \bar{F}_{B}^{P}(v, w), \bar{T}_{B}^{N}(v, w), \bar{I}_{B}^{N}(v, w), \bar{F}_{B}^{N}(v, w)\right)=(0,0,0,0,0,0)$

Hence $G=(A, B)$ is an isolated BSVNGs

Proposition 3.2: The notion of isolated BSVNGs generalized the notion of isolated fuzzy graphs.

Proof: If the value of $\mathrm{I}_{\mathrm{A}}^{\mathrm{P}}(w)=\mathrm{F}_{\mathrm{A}}^{\mathrm{P}}(w)=\mathrm{T}_{\mathrm{A}}^{\mathrm{n}}(w)=\mathrm{I}_{\mathrm{A}}^{\mathrm{n}}(w)=\mathrm{F}_{\mathrm{A}}^{\mathrm{n}}(w)=0$, then the notion of isolated BSVNGs is reduced to isolated fuzzy graphs.

Proposition 3.3: The notion of isolated BSVNGs generalized the notion of isolated SVNGs.

Proof: If the value of $\mathrm{T}_{\mathrm{A}}^{\mathrm{n}}(w)=\mathrm{I}_{\mathrm{A}}^{\mathrm{n}}(w)=\mathrm{F}_{\mathrm{A}}^{\mathrm{n}}(w)=0 \mathrm{I}_{\mathrm{A}}^{\mathrm{n}}(w)$, then the concept of isolated BSVNGs is reduced to isolated SVNGs.

\section{Conclusion}

In this article, we have extended the notion of isolated SVNGs to the notion of isolated BSVNGs. The notion of isolated BSVNGs generalized the isolated SVNGs. 


\section{References}

1. Smarandache, F: Neutrosophy, Neutrosophic Probability, Sets and Logic, Proquest Information \& Learning, Ann Arbor, Michigan, USA, 105p, (1998).

2. Atanassov, K.: Intuitionistic Fuzzy Sets. Fuzzy Sets and Systems, Vol.20, (1986) 87-96.

3. Zadeh, L.: Fuzzy sets. Information and Control, 8 (1965) 338-335.

4. Wang, H., Smarandache, F., Zhang Y., and Sunderraman, R.: Single Valued Neutrosophic Sets. Multispace and Multistructure 4 (2010) 410-413.

5. Deli, I., Ali, M., Smarandache, F.: Bipolar Neutrosophic Sets and Their Application Based on Multi-criteria Decision Making Problems, in: Advanced Mechatronic Systems (ICAMechS) (2015) 249-254.

6. http://fs.gallup.unm.edu/NSS/.

7. Smarandache, F.: Refined Literal Indeterminacy and the Multiplication Law of Sub Indeterminacies. Neutrosophic Sets and Systems, Vol.9, (2015) 58-63.

8. Gani, A., and Shajitha, B.S.: Degree: Order and size in Intuitionistic Fuzzy Graphs. International Journal of Algorithms, Computing and Mathematics, 3(3) (2010).

9. Akram, M.: Bipolar Fuzzy Graphs. Information Science, https://doi.org/10.1016/j.ins.2011. 07.037 (2011).

10. Bhattacharya, P.: Some Remarks on Fuzzy Graphs. Pattern Recognition Letters 6 (1987) 297-302.

11. Broumi, S., Talea, M., Bakali, A., Smarandache, F.: Single Valued Neutrosophic Graphs. Journal of New Theory, N 10, (2016) 86-101.

12. Broumi, S., Talea, M., Smarandache, F. and Bakali, A.: Single Valued Neutrosophic Graphs: Degree, Order and Size. IEEE International Conference on Fuzzy Systems (2016) 24442451.

13. Broumi, S., Bakali, A., Talea, M., Smarandache, F.: Isolated Single Valued Neutrosophic Graphs. Neutrosophic Sets and Systems, Vol.11 (2016) 74-78.

14. Broumi, S., Talea, M., Bakali, A., Smarandache, F.: On Bipolar Single Valued Neutrosphic Graphs. Journal of New Theory, N11 (2016) 84-102.

15. Broumi, S., Smarandache, F., Talea, M. and Bakali, A.: An Introduction to Bipolar Single Valued Neutrosophic Graph Theory. Applied Mechanics and Materials, vol 841, (2016) 184- 191.

16. Broumi, S., Bakali, A., Talea, M., Smarandache, F.: Generalized Bipolar Neutrosophic Graphs of Type 1, 20th International Conference on Information Fusion, Xi'an, China-July 10-13, (2017) 1714-1720.

17. Hassan, Ali., Malik, M.A., Broumi, S., Bakali, A., Talea, M., Smarandache, F.: Special types of bipolar single valued neutrosophic Graphs, Annals of Fuzzy Mathematics and Informatics, Vol.14, N 1, (2017) 55-73. 